\title{
A New Method of Generate the PWM Wave Using Analog
}

\author{
Huang Jian \\ Xijing University, Xi'an 710123, China
}

\section{Keywords: PWM; Triangular wave; Comparator}

\begin{abstract}
PWM is pulse width modulation, by adjusting the width of the output square wave, has been widely used in motor speed control, LED brightness adjust, rectifier, audio output etc. Now we always use software to product PWM, this method has some limitation, not meet high accurate, fast PWM wave produce. According to this, a new method of generate the PWM wave using analog circuits has been raised. A triangular waveform generator and a comparator was used to generate a PWM waveform, Duty cycle was inversely proportional to the input voltage. Error amplifier PWM waveforms use a negative feedback to ensure high accuracy and linearity of the output. Test results showed that this design could product fast, high-accuracy PWM waveform, its output fixed or change by special regular. This design produced a new idea for hardware engineer when they want to design different PWM waves, has a certain reference values.
\end{abstract}

\section{Introduction}

Pulse width modulation is an analog control method, usually use digital microprocessor output to control a very effective technique for analog circuits, is widely used in many fields of measurement, communication and power control and conversion. Pulse width modulation is a very effective technique to control the analog circuit by digital output of microprocessor. PWM control technology is the most widely used control mode of power electronic technology with the advantages of simple control, flexible and dynamic response, and it is also a hot spot for people to study. Combining with the modern control theory or realizing the non-resonant wave switching technology will become one of the main directions of the development of PWM control technology.

Based on research the principle of PWM, put forward the PWM wave produced by high performance analog design, with a triangular waveform generator and a comparator to generate a pulse width modulation (PWM) waveform, this time for duty ratio is inversely proportional to the input voltage. An operational amplifier and a comparator generate a triangular waveform, which is input from the inverting input of the second comparator. Input voltage through the comparator forward input, compared to produce a PWM waveform. A negative feedback on the PWM waveform of the error amplifier is used to ensure the high accuracy and linearity of the output [1-3].

\section{System Design}

The system block diagram is shown in Fig. 1, the input voltage signal is input to the error amplifier circuit, and then the input to the comparator, and its negative feedback to the error amplifier circuit, to improve the accuracy of the input signal. The triangular wave circuit generates a triangular wave and input to the comparator, and the input voltage of the triangular wave voltage and the error amplification circuit is compared by comparing the circuit, and the output of the PWM wave is generated. Change the input voltage signal, will produce different PWM waveform. By reasonable selection of high performance devices, the frequency and driving ability of wave can be improved PWM [4-6]. 


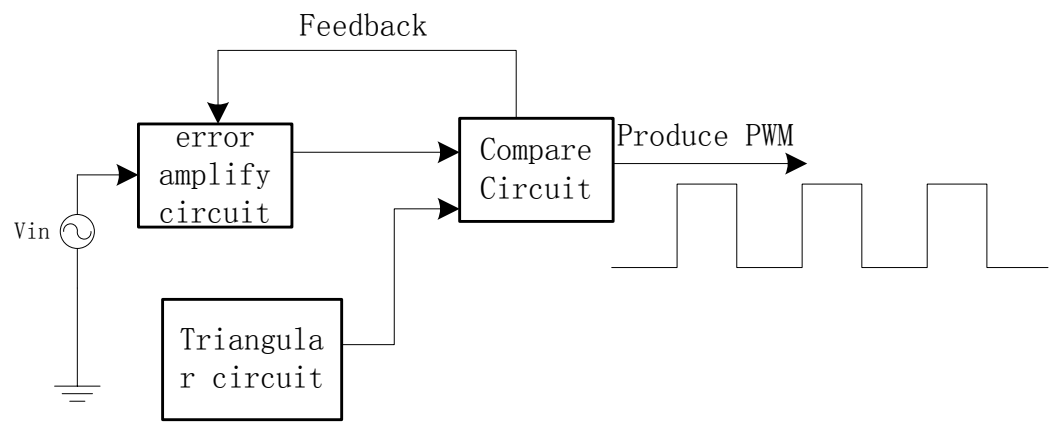

Figure 1. System design diagram

\section{Detailed Design and Key Technologies}

Error Amplifier Circuit Design. The error amplifier circuit is shown in Fig. 2. There are two purposes, first, in order to correct the output voltage error introduced by the comparator, the error amplifier to adjust the output PWM waveform feedback. Second, it adds a DC offset to the input voltage, so that the circuit can be adjusted by the negative input voltage. $2.2 \mathrm{~V}, 50 \mathrm{MHz}$ low noise single supply High Performance CMOS rail to rail operational amplifier is OPA365. After optimization has very low voltage, low noise and high speed operation (50 MHz gain bandwidth) and excellent common mode suppression ratio (CMRR), no input stage cross-over distortion, high output impedance and rail to rail input and output swing. Input common mode range includes negative and positive power supply. Output voltage swing is $10 \mathrm{mV}$. Suitable for driving sampling analog to digital converter (ADC), support for audio, signal conditioning, sensor amplification, national defense, aerospace and medical applications. It is also very suitable for the application of mobile power amplifier control loop.

Most of the PWM circuits are not running the comparator included in the feedback loop of an amplifier. For these circuits, the output duty cycle is a function of the amplitude of the triangular wave (Vref) and the input signal amplitude (Vin). The average output voltage (Vo) depends on the amplitude of the PWM waveform (Vpwm), which is usually determined by the power supply voltage of the comparator. Voltage (Vo) output as shown in the formula (1):

$$
V_{O}=K \times V_{P W M}=\frac{V_{T R I}+V_{I N}}{2 \times V_{T R I}} \times V_{P W M}
$$

Since the power supply voltage of the comparator directly affects the output voltage, the non-feedback PWM circuit has no power supply rejection. In this design, an error amplifier is operated as an input signal of an inverting amplifier, which is shown as a DC coupled source V input. By incorporating the comparator in the feedback loop of the error amplifier, and adding an integrated capacitor $\mathrm{C} 2$, the error amplifier directly controls the average output voltage [7]. Voltage (Vo) output as shown in the formula (2):

$$
V_{O}=\frac{R_{4}}{R_{3}} \times V_{I N}+V_{R E F}
$$

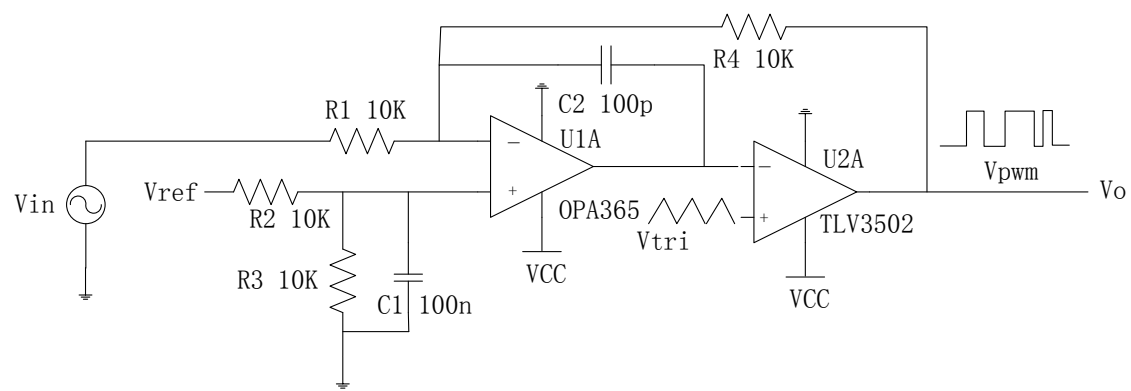

Figure 2. Error amplifier circuit

Triangle Wave Generator Circuit Design. Triangular wave generator as shown in Fig. 4, operational amplifier is configured to an integrator, and its output in the output of the comparator is 
a low electricity at ordinary times increase linearly. On the contrary, when the comparator output is high, the output is reduced linearly. When the non-inverting input voltage exceeds the reference voltage, the comparator output switches, in this circuit, the reference voltage is $2.5 \mathrm{~V}$. Since the circuit is designed to supply power to a single $2.5 \mathrm{~V}$, a $5 \mathrm{~V}$ offset is added to the triangular wave, so that it is centered on the power point of all the comparator in the circuit. The amplitude of the triangular wave is generated by the formula (3).

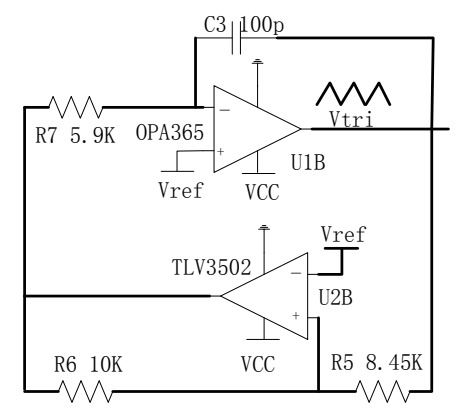

Figure 4. Triangle wave generator circuit design

$$
V_{\text {ref }}=\left(\frac{R_{5}}{R_{6}}\right) V_{1}
$$

In the formula (4), the V1 is higher or lower than the reference voltage of the comparator output voltage. For example, in our system, the comparator output will be $5 \mathrm{~V}$ or OV. Because this circuit uses a $2.5 \mathrm{~V}$ reference, VI is equal to $2.5 \mathrm{~V}$. Most PWM systems do not allow for $0 \%$ or $100 \%$ modulation, this is because the output will be $\mathrm{DC}$, and there may be damage to other components in the signal path. Therefore, the amplitude of the triangular wave must be chosen so that it is greater than the maximum expected input voltage. Selection of $2.1 \mathrm{~V}$ through the comparison can be achieved $2 \mathrm{~V}$ to $+2 \mathrm{~V}$ voltage range. Select $\mathrm{R} 6$ for the $10 \mathrm{~K}$ resistance, through the formula (4) can be regarded as R5:

$$
R_{5}=\frac{V_{T R I} \times R_{6}}{V_{1}}=\frac{2.1 \mathrm{~V} \times 10 \mathrm{~K}}{2.5 \mathrm{~V}}=8.45 \mathrm{~K}
$$

Generation of PWM Wave. The output of the U1B in the triangular wave generator circuit in Fig. 4 is connected to the positive input of the error amplifier circuit U2A comparator. After comparison with the input voltage, the PWM wave can be output at the output end of the U2A. The output voltage of the Vo is calculated by the formula (5).

$$
V_{o}=K \times V_{P W M}
$$

Among them, Vo is the average output voltage, $\mathrm{K}$ is the duty cycle of the PWM waveform, and Vpwm is its amplitude. For example, a duty cycle of 25\% PWM 5V waveform will produce a $1.25 \mathrm{~V}$ output voltage at low pass filter.

\section{Test Results}

According to the hardware design, the circuit is simulated in TINA-TI, and the following data and simulation waveforms are available. When the input voltage is fixed value, the waveform is generated when the input voltage is $3 \mathrm{~V}$. As can be seen from the figure, the duty cycle of the PWM is proportional to the lower half of the triangle wave, and the duty cycle of the output PWM can be adjusted by adjusting the input DC voltage. 


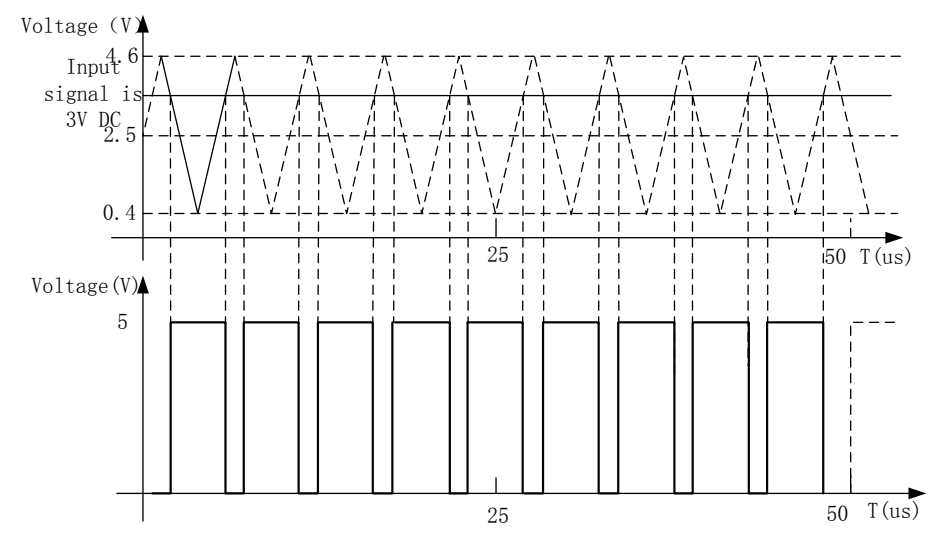

Figure 5. The output PWM waveform when input signal is $3 \mathrm{~V} \mathrm{DC}$

\section{Conclusion}

A new method of generate PWM with high performance analog parts is produce, and we build the hardware circuit, careful analysis the principle, simulate the result under the TINA-TI simulation. The test result show that can generate different duty ratio PWM wave according to different input signal. The design objectives and test results can meet the requirements of the basic requirements. It also can meet the specific needs of PWM wave generation by adjusting the parameters of the hardware circuit, or using different devices. The hardware designers have a certain reference and practical value.

\section{References}

[1] Texas Instruments. OPA365 [M].Shang Hai, 2009, 9.

[2] Du Shaotong, Wu Xiaojie, Zhou Juan, Wang Fujun, Yang Yu. A new PWM algorithm based on virtual flux model for prediction of [J], China Electrical Engineering Chinese Journal, 2015,35 (3): $688-693$

[3] Fan Weijun, Zhang Hao, Yu Guiying. Design of Jing Shi Shangguan, [J] PWM digital adjustable uniform light source, liquid crystal display. 2013,30 (1): 88-94

[4] Zhao Kuiyin. Fuzzy sliding mode variable structure control for PWM rectifier [J], Proceedings of the Chinese society of electrical engineering, 2006,21 (7): 49-53

[5] Liubo and Ben Hongqi, Meng Tao. The Z source PWM rectifier DC side control strategy [J], Proceedings of the CSEE, 2015,19 (1): 16-22

[6] Gu Liang, Mao Jingfeng, Cheng Ying, Zhao Suhua. Distributed wireless intelligent LED illumination control system [J]. Computer measurement and control, 2015, 23 (1) 115-117131.

[7] Liu Lijuan, Fu Zhizhong, Zhang Peiming, Chen Zhenglong. Design of high color rendering index LED driving circuit [J], power technology.2015, 39 (2):357-359.

[8] Zhang Yongchang, Xie Wei, Li Zhengxi. Research on power pulse minimization method for PWM rectifier [J], Proceedings of the Chinese society of electrical engineering.2013,33 (18):57-63

[9] Zhou Meilan, Gao Zhaoming, Wu Xiaogang, Li Zhi. Effects of five PWM modes on commutation torque ripple in Brushless DC motor system [J], Journal of motor and control.2013,17 (7): 15-21

[10]Cao Xiaodong, Tan Guojun, Wang Conggang, Li Hao. A satisfactory predictive control strategy for a low switching frequency PWM rectifier [J], Proceedings of the Chinese society of electrical engineering.2013,33 (27): 69-77 